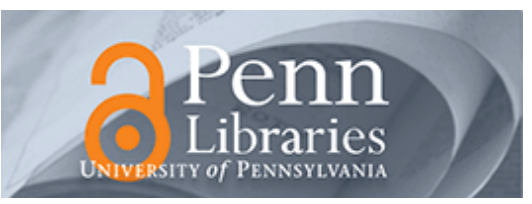

University of Pennsylvania

ScholarlyCommons

Departmental Papers (BE)

Department of Bioengineering

January 2008

\title{
Integrin-mediated signalling through the MAP-kinase pathway
}

\author{
Ka Lai Yee \\ University of Pennsylvania
}

V. M. Weaver

University of California

Daniel A. Hammer

University of Pennsylvania, hammer@seas.upenn.edu

Follow this and additional works at: https://repository.upenn.edu/be_papers

\section{Recommended Citation}

Yee, K. L., Weaver, V. M., \& Hammer, D. A. (2008). Integrin-mediated signalling through the MAP-kinase pathway. Retrieved from https://repository.upenn.edu/be_papers/104

Copyright 2008 IEEE. Reprinted from IET Systems Biology, Volume 2, Issue 1, January 2008, pages 8-15.

This material is posted here with permission of the IEEE. Such permission of the IEEE does not in any way imply IEEE endorsement of any of the University of Pennsylvania's products or services. Internal or personal use of this material is permitted. However, permission to reprint/republish this material for advertising or promotional purposes or for creating new collective works for resale or redistribution must be obtained from the IEEE by writing to pubs-permissions@ieee.org. By choosing to view this document, you agree to all provisions of the copyright laws protecting it.

This paper is posted at ScholarlyCommons. https://repository.upenn.edu/be_papers/104

For more information, please contact repository@pobox.upenn.edu. 


\title{
Integrin-mediated signalling through the MAP-kinase pathway
}

\author{
Abstract \\ The mitogen activated protein (MAP) kinase cascade, leading to extracellular-regulated kinase (ERK) \\ activation, is a key regulator of cell growth and proliferation. The effects of ERK are mediated by \\ differences in ERK signalling dynamics, including magnitude and duration. In vivo, ERK signalling is \\ stimulated by both growth factors and adhesion signals. A model for adhesionmediated ERK activation is \\ presented. Outputs of the model such as ERK and FAK activation, as well as responses to different ligand \\ densities, are compared with published experimental data. The model then serves as a basis for \\ understanding how adhesion may contribute to ERK signalling through changes in the dynamics of focal \\ adhesion kinase activation. The main parameters influencing ERK are determined through screening \\ analyses and parameter variation. With these parameters, key points in the pathway that give rise to \\ changes in downstream signalling dynamics are identified. In particular, oncogenic Raf and Ras promote \\ cell growth by increasing the magnitude and duration, respectively, of ERK activity.

\section{Comments} \\ Copyright 2008 IEEE. Reprinted from IET Systems Biology, Volume 2, Issue 1, January 2008, pages 8-15. \\ This material is posted here with permission of the IEEE. Such permission of the IEEE does not in any way \\ imply IEEE endorsement of any of the University of Pennsylvania's products or services. Internal or \\ personal use of this material is permitted. However, permission to reprint/republish this material for \\ advertising or promotional purposes or for creating new collective works for resale or redistribution must \\ be obtained from the IEEE by writing to pubs-permissions@ieee.org. By choosing to view this document, \\ you agree to all provisions of the copyright laws protecting it.
}




\title{
Integrin-mediated signalling through the MAP-kinase pathway
}

\author{
K.L. Yee, V.M. Weaver and D.A. Hammer
}

\begin{abstract}
The mitogen activated protein (MAP) kinase cascade, leading to extracellular-regulated kinase (ERK) activation, is a key regulator of cell growth and proliferation. The effects of ERK are mediated by differences in ERK signalling dynamics, including magnitude and duration. In vivo, ERK signalling is stimulated by both growth factors and adhesion signals. A model for adhesionmediated ERK activation is presented. Outputs of the model such as ERK and FAK activation, as well as responses to different ligand densities, are compared with published experimental data. The model then serves as a basis for understanding how adhesion may contribute to ERK signalling through changes in the dynamics of focal adhesion kinase activation. The main parameters influencing ERK are determined through screening analyses and parameter variation. With these parameters, key points in the pathway that give rise to changes in downstream signalling dynamics are identified. In particular, oncogenic Raf and Ras promote cell growth by increasing the magnitude and duration, respectively, of ERK activity.
\end{abstract}

\section{Introduction}

The MAP kinase cascade is an important signalling pathway associated with tumorigenic behaviour. In cancerous cells, the pathways that regulate proliferation are disrupted so that cells experience unchecked growth. A major factor in proliferation is the increased activity of the MAPK cascade, which can be caused by increased external stimuli or mutations along the pathway that promote pathway activation [1]. The MAPK involved in growth regulation is extracellular-regulated kinase (ERK). Upon double phosphorylation by mitogen activated protein kinase (MEK), double phosphorylated extracellular-regulated kinase (ERKPP) translocates to the nucleus and induces gene transcription by phosphorylation of transcription factors [2]. One of ERK's targets is fos, a member of the AP-1 transcription factor heterodimer [3]. AP-1 family transcription factors induce the transcription of cyclin D1, which promotes cell-cycle entry [4, 5]. Modelling this pathway to gain an understanding of how specific elements give rise to different ERK signalling dynamics is an important step towards regulating signal transduction, and ultimately cell behaviour, in vivo.

The signalling pathway that culminates in ERK activation is initiated by extracellular stimuli such as adhesion to the extracellular matrix or growth factors. Cell adhesion activates ERK by binding of $\alpha 5 \beta 1$ integrins at the cell surface to extracellular matrix proteins such as fibronectin $[6,7]$. Integrins activate ERK via a pathway involving Shc, Grb2, Sos, the GTPase Ras and focal adhesion

(C) The Institution of Engineering and Technology 2008

doi:10.1049/iet-syb:20060058

Paper first received 4th August 2006 and in revised form 16th April 2007

D.A. Hammer and K.L. Yee are with the Department of Bioengineering, University of Pennsylvania, 240 Skirkanich Hall, 210 S 33rd St, Philadelphia PA 19104, USA

V.M. Weaver is with the Department of Surgery, University of California San Francisco (UCSF), 513 Parnassus Ave, Room S-321, San Francisco, CA 94143-0470, USA

E-mail: hammer@seas.upenn.edu kinase (FAK) [5, 6, 8]. Epidermal growth factor (EGF) binding to its receptor is also known to activate ERK through the same Shc-Grb2-Sos-Ras pathway as integrins [9-11]. Although integrins may activate ERK in the absence of growth factors, cells in vivo are exposed to both adhesion signals and growth factor signals. In addition, there is a cross-talk between growth factors and integrin-activated pathways to ERK activation at different levels of the pathway [12-14]. This cross-talk likely gives rise to distinctive ERK activation signals that can regulate different behaviours [12]. For example, ERK activity stimulated by growth factors and adhesion independently is transient, whereas ERK activity in adherent, growth factor stimulated cells is sustained. Moreover, the cell-cycle protein cyclin D1 is only induced by sustained ERK signals $[5,15]$.

The MAPK cascade and activation by EGF have been previously explored with deterministic, computational models. A basic model of the Shc-Grb2-Sos-Ras pathway activated by EGF was described by Kholodenko et al. [16]. A more comprehensive model of ERK activation in response to EGF stimulation has also been examined [17]. In that model, EGF receptor-ligand binding and dimerisation initiate the traditional Shc-Grb2-Sos-Ras pathway that leads to the MAPK cascade. More recently, Chapman and Asthagiri [18] undertook an exploration of MAPK cascade dynamics. Their work demonstrated that adjusting rate constants and protein or phosphatase concentrations controls ERK activation dynamics. Similarly, Hornberg et al. [19] applied control analysis to the model developed by Schoeberl, identifying specific proteins that are important in EGF-mediated ERK signalling.

As a first step towards developing a model of ERK activation by both EGF and integrins, a computational model for integrin activation of ERK is presented in this paper. In this model, the ERK signalling pathway is initiated by $\alpha 5 \beta 1$ integrin binding to its extracellular ligand fibronectin. The model successfully simulates experimental FAK and ERK activation time courses. Semi-quantitative data on FAK and ERK activation after adhesion [20] are used to 
verify the results of the model. After comparing simulated signals with experimental results, the pathways were explored to gain insight into specific characteristics that contribute to ERK activation dynamics. To systematically explore the set of parameters used in the model, a screening method developed by Morris [21] was used to identify the key parameters that affect different characteristics of the output signal, such as overall dynamics, the maximum value and time to reach the maximum.

\section{Methodology}

\subsection{Biological considerations}

The signalling events culminating in ERK activation are modelled as a set of differential equations. The proteinprotein interactions included in the pathway were chosen based on published pathways and experimental data and are illustrated in Fig. 1 $a$. The full pathway and a list of reactions and rate constants are provided in the Supplementary Materials.

The integrin/FAK activation portion of the model deals with receptor-ligand binding as a cell spreads on fibronectin. The cell-surface contact area begins at zero and increases according to an experimentally determined profile [22] (see Supplementary Materials). The increase in contact area regulates the transport of receptors and ligands into the contact
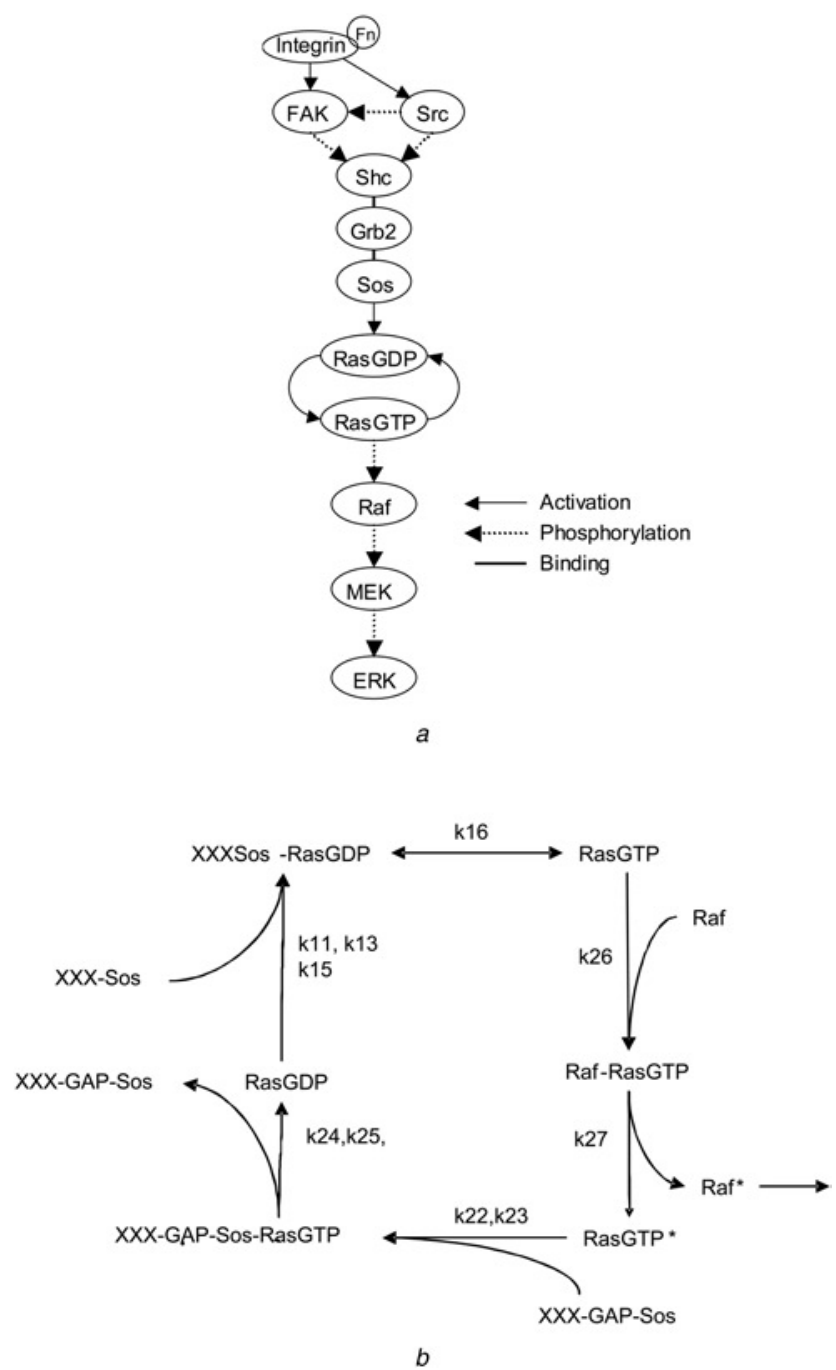

Fig. 1 Protein-Protein Interactions

a Signalling pathways for ERK activation by adhesion $b$ Ras cycle of GDP/GTP binding area where binding occurs. Integrin-fibronectin binding is modelled according to the Bell model for receptor-ligand binding [23]. Because integrin clustering is a complex process involving the many cytoskeletal proteins and the transduction for force, clustering is modelled as dimerisation of receptor-ligand pairs (see Supplementary Materials). We assume that only dimerised receptor-ligand pairs are able to recruit FAK, which becomes autophosphorylated at tyrosine 397 (FAKP) [24].

Src activation occurs after cell adhesion, although it is unclear how adhesion signals regulate the Src activation machinery [25]. Activation begins with the dephosphorylation of cytoplasmic Src at tyrosine 527. The protein then undergoes a conformational change and translocates to the membrane [26]. To be fully active, Src undergoes an intermolecular autophosphorylation at Y416. However, fully active Src can be re-phosphorylated and dephosphorylated, thereby cycling it from active to inactive conformations [27-29]. This module is linked to the rest of the pathway by the ability of Src to phosphorylate its substrate FAK at Y925 [30]. Both Src and FAK are capable of phosphorylating Shc $[9,11,31]$. This interaction leads to the same sequence of signalling events the activated by the EGF receptor, ultimately resulting in the activation of the MAPK cascade $[32,33]$.

Evidence suggests that integrins may also activate ERK through a FAK-independent pathway [9]. Such a pathway likely involves Fyn, a member of the Src kinase family, and the alpha integrin subunit [34]. Because of limited experimental data on this pathway, this model focuses on the FAK-dependent pathways to ERK activation.

\subsection{Model parameters}

Rate constants and protein concentrations were either found in literature or estimated based on known constants for similar reactions. For protein interactions without published binding constants, $K_{\mathrm{d}}, k_{\mathrm{f}}$, and $k_{\mathrm{r}}$ were estimated from available peptide binding data. For example, binding constants for Src and FAKP in the Src activation module were estimated with rate constants for Src SH2 domain binding to the pYEEI peptide, which is similar to the autophosphorylation site for FAK $[35,36]$. Most binding events in this pathway involve $\mathrm{SH} 2-$ phosphotyrosine and SH3-PXXP binding [37, 38].

During integrin activation of FAK, $k_{1}$ is the rate of dimerisation of receptor-ligand pairs, whereas $k_{\mathrm{p}}$ and $k_{\mathrm{m}}$ are the forward and backward rate constants for FAK binding to receptor-ligand dimers and subsequent autophosphorylation. $k_{1}$ is a phenomenological rate constant assigned a value of $0.01 \mathrm{~s}^{-1}$, which corresponds to maximum levels of FAK activation. Decreasing $k_{1}$ will decrease the final magnitude of FAK activation without significantly changing the kinetics. Values for $k_{\mathrm{p}}$ and $k_{\mathrm{m}}$ were estimated to obtain a variety of time scales for FAK autophosphorylation, which are discussed in Section 3. The Src activation module also contains the phenomenological rate constant $k_{\mathrm{t}}$, which represents the rate of Src transport from the cytoplasm to the membrane by the cytoskeleton [39]. Because these steps are not easily modelled, they are lumped into the transport rate $k_{\mathrm{t}} ; k_{\mathrm{t}}$ is set at $0.1 \mathrm{~s}^{-1}$, at which the rate of cytoskeletal transport is maximal. Rate constants for the phosphatase and kinase reactions in Src activation were obtained from literature [40-42]. Subsequent analyses of FAK and downstream signalling focus on the relative effects of different parameter values on kinetics and magnitude, so that the conclusions drawn are valid regardless of the specific phenomenological constants used. 
Initial protein concentrations were obtained from Schoeberl et al. [17] and were varied over several orders of magnitude to explore their effects on signal dynamics. The constant concentrations of phosphatases were also estimated based on these initial values. The initial conditions specify protein concentrations for FAK, Src, Shc, Grb2, Sos, MEK and ERK. All other protein species present in the system at any given time are derived from these proteins and have an initial concentration of zero.

The resulting set of equations with initial conditions was solved in MATLAB 6.5 using the ODE15s solver.

\section{Results}

\subsection{Kinetics of FAK and ERK activation}

Asthagiri et al. [20] have shown that activation of ERK by adhesion occurs before maximal FAK activation. Simulations of ERK and FAK activation showed similar kinetics (Fig. 2), which was also robust over large ranges of parameter values (see Section 3.4.2). Over a range of FAK recruitment rates $\left(k_{\mathrm{p}}\right)$, the ERK activation peak consistently occurs ahead of full FAK activation. Since FAK is the only means by which ERK is activated in this model, early and therefore relatively low levels of FAK activation are sufficient for stimulating the MAPK cascade. For FAK to achieve sufficient levels of activation to stimulate the MAPK cascade at early times, it was found that the total level of FAK must be higher than that of downstream effectors such as Sos, the activator of the MAPK cascade. For example, a peak magnitude of $1.4 \mathrm{nM}$ ERKPP at $t=2800 \mathrm{~s}$ requires the amount of total FAK to be nearly 22 times the amount of Sos. As a result, it is assumed that the amount of FAK is in excess of other signalling proteins in the system. Lastly, sustained FAK activation does not necessarily result in sustained ERK activation, implying that FAK serves as an initial stimulus for ERK activation, but the duration of ERK activation is also regulated by downstream factors that will be discussed.

\subsection{FAK and ERK activation in response to ligand density}

Fibronectin density is one of the most readily controllable experimental parameters in the system and has been shown to affect both the magnitude and kinetics of ERK

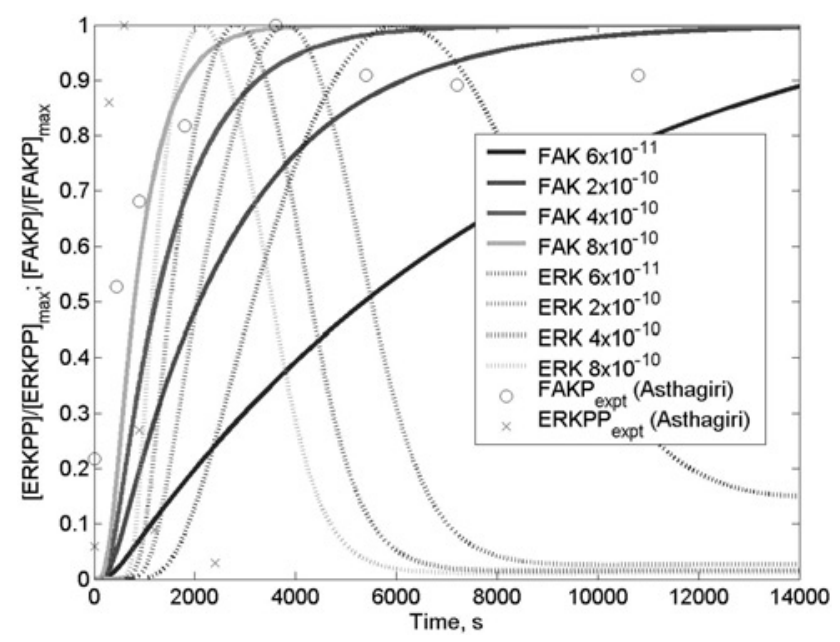

Fig. 2 FAK and ERK activation over a range of $k_{\mathrm{p}}$, assuming that $K_{\mathrm{d}}=k_{m} / k_{p}=1066$ is constant

Experimentally observed time courses (Asthagiri) are also plotted activation [20]. As ligand density increases, the magnitude of the ERKPP peak also increases, whereas the peak occurs faster. The effects of ligand density were successfully replicated in silico for similar fibronectin densities, assuming a high receptor density of $7.89 \times 10^{10}$ molecules $/ \mathrm{cm}^{2}\left(\sim 1.5 \times 10^{6} /\right.$ cell) (Fig. $\left.3 a\right)$. Not surprisingly, changes in ligand density had substantial effects on ERKPP dynamics only when ligand density is nonsaturating $\left(R_{\mathrm{t}}>L_{\mathrm{t}}\right)$, thus requiring a high assumed receptor density. Furthermore, the linear correlation between the ligand density and initial rates of FAK and ERK activation observed by Asthagiri et al. [20] was also seen in simulations (Fig. 3b). At saturating ligand densities, the linear correlation fails.

\subsection{Exploration of signalling dynamics}

\subsubsection{Relationship between FAK and ERK activation:}

The effects of ligand density on ERK raise the question of how the signal is transduced downstream. Since FAK is a major mediator between adhesion-based signals and downstream signals, it is reasonable to examine the effects of
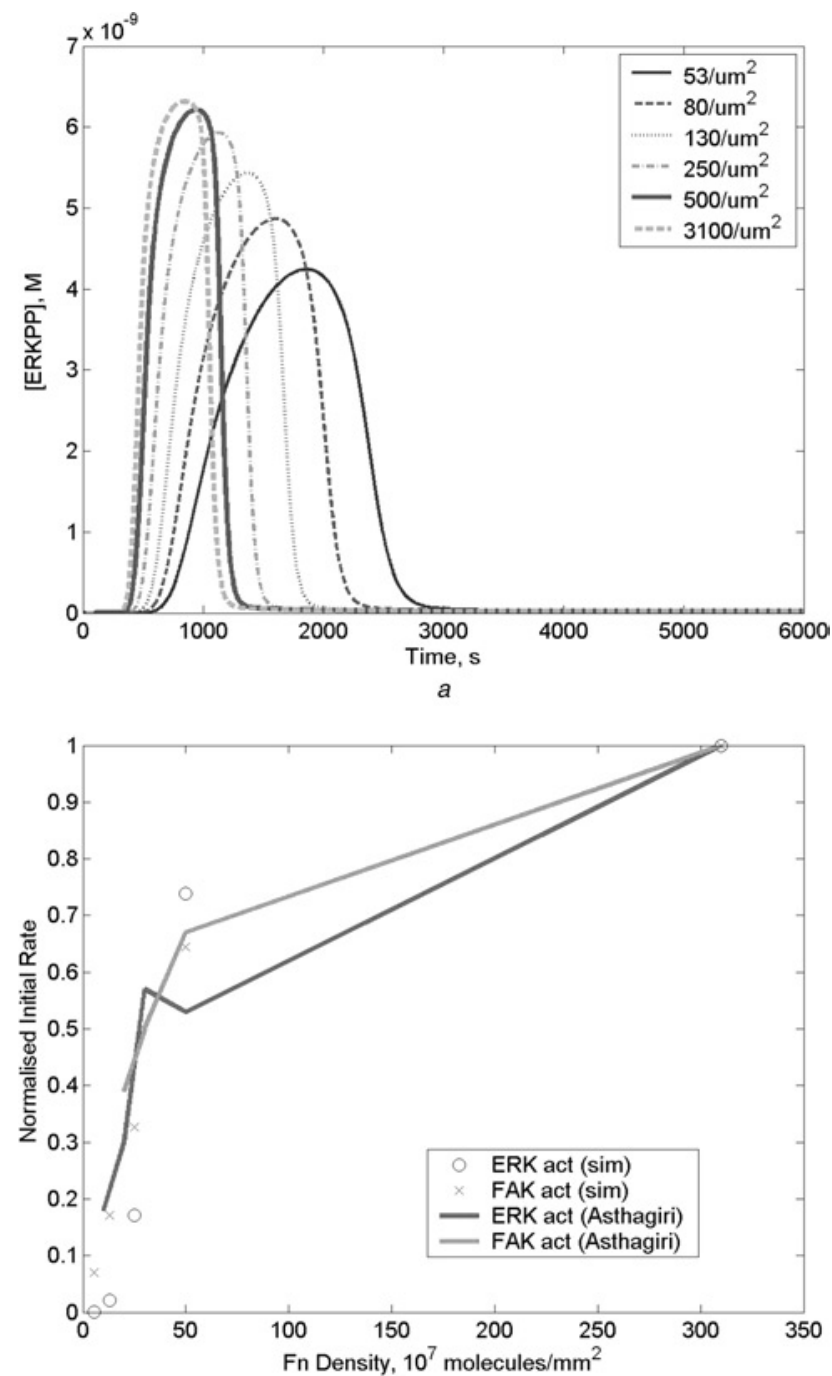

$b$

Fig. 3 Effects of ligand density

$a$ ERK activation in response to changes in fibronectin density $b$ Initial rates of FAK and ERK activation with ligand density Initial rates were calculated from 470 to $480 \mathrm{~s}$, which falls within the linear phase of activation

Onset of the linear phase is subject to a time lag due to the clustering process 
ligand density on FAK activation to understand how such changes can affect ERK. Because FAK is in excess of ligand and receptor binding sites, the magnitude of FAKP is directly proportional to ligand density, whereas the time required to reach the maximal FAKP is unchanged. As shown in Fig. $3 a$, increases in ligand density correspond to increases in ERKPP magnitude and decreases in the time to reach the maximum. To determine whether such effects are specific to changes in the magnitude of FAKP, we considered the effects of changes in the time to reach the maximal FAKP by changing the total amount of FAK. Increased levels of FAK drive the rate of its activation, resulting in increased kinetics without changing the magnitude of FAKP. Fig. $4 a$ shows that equivalent increases in ligand density and FAK decrease the time of the ERKPP peak similarly. Fig. $4 a$ also illustrates that ligand density has a more pronounced effect on ERKPP magnitude than does FAK. In order to better compare the effects of magnitude and kinetics directly, FAK activation dynamics are
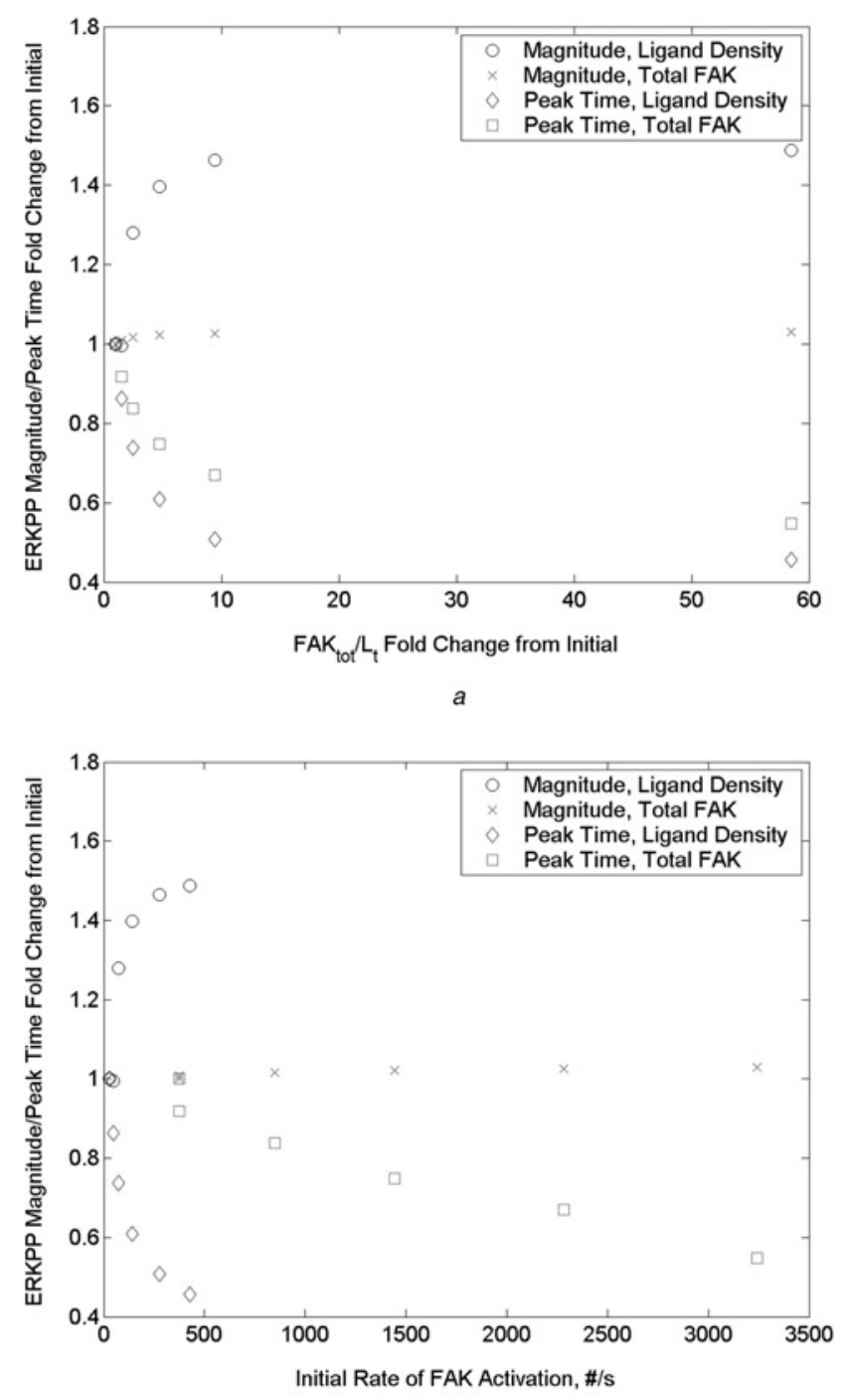

b

Fig. 4 Effect of increase in ligand density and FAK on time

a Fold changes in ERK activation dynamics as a function of fold changes in ligand density and total FAK

All fold changes are normalised to the starting values $\left(53 / \mu \mathrm{m}^{2}\right.$ and $2 \times 10^{6}$, respectively)

$b$ Fold changes in ERK activation dynamics as a function of the initial rate of FAK activation

Initial rates in response to changes in ligand density and total FAK were calculated based on the dynamics of FAK activation from $t=470-480 \mathrm{~s}$, where the signal is linear expressed as an initial rate of activation (Fig. $4 b$ ). If the effects of FAKP magnitude and kinetics are equivalent, each initial rate calculated from either ligand density or total FAK changes should correspond to a unique change in ERK activation dynamics. However, the curves for ERKPP dynamics differ depending on ligand density or total FAK changes, indicating that the effects are parameterspecific. In fact, ligand density, via changes in the magnitude of FAKP, appears to be a stronger regulator of ERKPP dynamics than total FAK, which changes the kinetics of FAKP. Furthermore, the differing curves in response to ligand density and FAK imply that the initial rate of FAKP is not the sole determinant of ERKPP dynamics and that other factors affected by these two parameters are also playing a role. Moreover, FAK-independent pathways are not likely the source of these factors because their contributions to ERK activation are only on the order of $10^{-19}$ to $10^{-18} \mathrm{M}$. Regardless of these factors, the relationship between ERKPP and initial rate is nonlinear and plateaus as the initial rate in creases. Thus, the effects of ligand density and FAK will attenuate as these two factors reach saturation.

\subsubsection{Ras and Raf as regulators of ERK activity: To} better understand the behaviour of the system, parameters that play a key role in regulating the dynamics of the ERK signal were identified by parameter variations. The duration of ERK activation is found to be particularly sensitive to the concentrations of Ras and Raf. Testing Raf concentrations over an order of magnitude from 10 to $100 \mathrm{nM}$ produced ERKPP signals with a range of durations (Fig. 5a). At or above a [Raf] of $100 \mathrm{nM}$, the ERKPP signal is a sharp peak at approximately 5 min, which falls off quickly by $30 \mathrm{~min}$. As [Raf] is decreased, the ERKPP signal duration increases until a sustained signal is obtained at $10 \mathrm{nM}$. Between 20 and $60 \mathrm{nM}$, very small changes in Raf concentration can give rise to significant changes in signal duration. For example, a 25\% concentration decrease from 40 to $30 \mathrm{nM}$ results in a signal duration increase of nearly $45 \%$. For high [Raf], the ERKPP signal is very similar to that obtained by Asthagiri et al. [20]. Similarly, the duration of the ERKPP signal increases with increasing levels of Ras (Fig. 5b). Although the changes in ERKPP are not as sensitive to small changes in [Ras], large increases in [Ras] do yield significant prolongation of ERK activation.

The sensitivity of ERKPP to Raf and Ras originates at the locus of intersection between the RasGTP/GDP cycle and the MAPK cascade (Fig. 1b), where RasGTP binds and activates Raf, forming Raf*. The fast drop off in signalling that forms the peak in ERKPP signals results from the depletion of RasGTP available for interaction with Raf. When RasGTP is depleted, no more Raf can be activated causing the Raf* and subsequently ERKPP signals to fall off, resulting in the formation of a peak. Therefore, as [Raf] increases, the Raf-RasGTP binding reaction also becomes faster, thereby increasing the rate at which RasGTP is depleted. Similarly, Sydor et al. [43] have shown that the Raf-Ras complex has an extremely short lifetime, after which Raf is released for downstream signalling. The resulting Ras species, denoted RasGTP*, is no longer capable of activating Raf and will undergo GTP hydrolysis catalysed by GTpase activating proteins (GAPs), which are recruited to focal adhesions after integrin-ligand binding [44, 45]. In the case of high [Raf], Ras accumulates as RasGTP*, suggesting that the RafRasGTP reaction is much faster than the action of GAPs in recycling Ras. Thus, nearly $80 \%$ of Ras accumulates in the GTP* form and is not recycled fast enough to the 


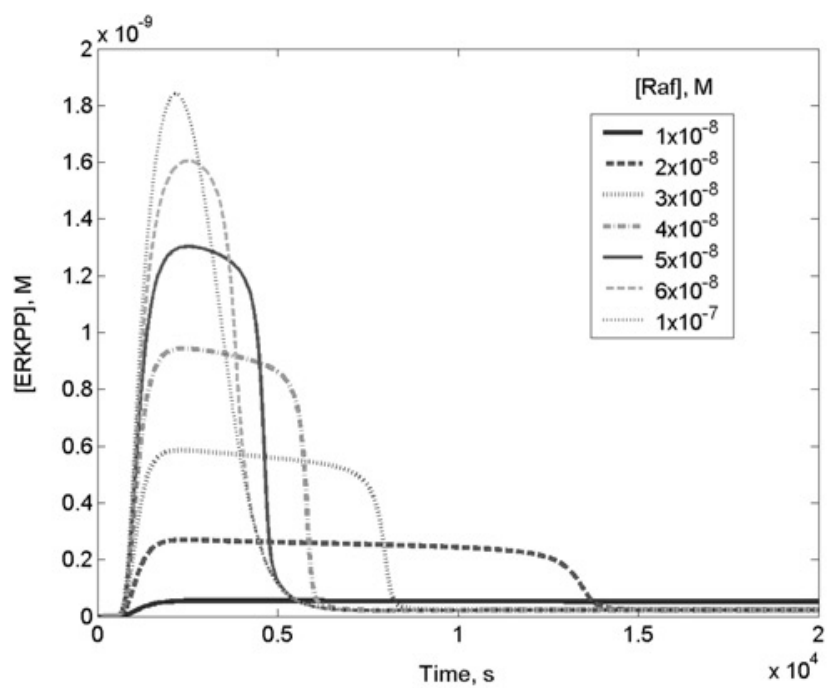

a

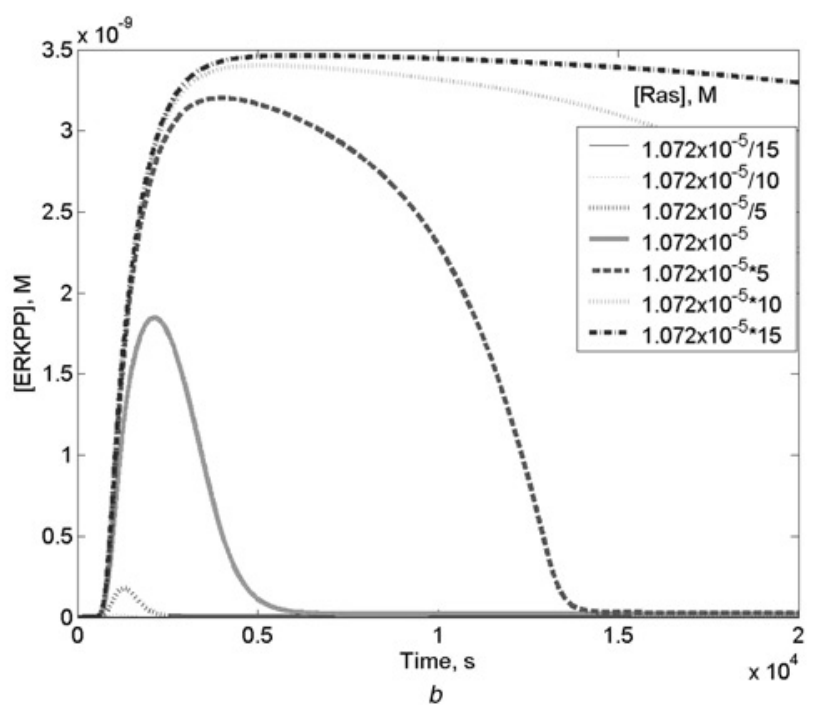

Fig. 5 ERK signals with a range of durations

$a$ ERK activation in response to [Raf]

[Ras] is assumed to be $1.072 \times 10^{-5} \mathrm{M}$

$b$ ERK activation in response to [Ras]

Raf is assumed to be $3.76 \times 10^{-7} \mathrm{M}$

RasGTP form to prevent depletion. As [Raf] is reduced, the rate of RasGTP conversion to RasGTP* is more comparable to the rate of recycling to RasGTP. Depletion of RasGTP is delayed and an increasingly sustained ERKPP signal is obtained. An increase in [Ras], conversely, increases the amount of RasGTP available, prolonging the ERKPP signal.

\subsection{Sensitivity analysis}

3.4.1 Morris screening: To systematically identify key parameters involved in ERK signalling, the Morris screening method [21] was applied to the model. The Morris method allows for the effects of a particular parameter variation to be seen in the context of different values for every other parameter, as opposed to fixing all other parameters. The result of the model is a measure of the effect each parameter has on the output signal. For the dynamics of ERK activation, the effect of each parameter on the overall ERK time course as measured by the norm of the change in output, time to reach the maximum, the peak magnitude and three specific time points was calculated. These values were normalised by the relative change in input values. However, because parameters were sampled over a log uniform distribution, the fractional change remains constant between parameters. Morris runs were performed until at least the top $20 \%$ of parameters converged $(n=160)$.

The main parameters involved in regulating ERK activation are rate constants associated with the MAPK cascade. However, of the factors that could be readily manipulated experimentally, Ras and Raf fall within the top $15 \%$ of the key parameters for several measures of ERK dynamics, confirming the effects on ERKPP signal duration described above (Table 1). Out of 106 parameters, Ras ranked second in importance for the time of the ERKPP peak, 16th based on overall time course and 29th based on a late time point that indicates signal duration. Similarly, Raf ranks fourth in determining the time of the ERKPP peak and seventh in early activation. Both Ras and Raf also fall within the top $20 \%$ of the parameters affecting the magnitude of ERK activation, ranking 18th and 19th, respectively. Interestingly, RasGAP ranks first out of all the parameters for the time of the ERK activation peak, again highlighting the importance of the RasGDP/GTP cycle in regulating ERK activation dynamics. Receptor density also falls within the top $15 \%$ of the key parameters affecting the ERKPP signal. Moreover, integrin density and contact area, which determine the total amount of fibronectin available, are ranked seventh and fiveth, respectively, as regulators of the kinetics of ERK activation. FAK is also identified as a key parameter in ERK activation kinetics, supporting the idea that initial FAK signalling contributes significantly to the activation of ERK. Integrin density, and not fibronectin density, was likely identified in the Morris screening because of the relative values of the two parameters used. Depending on the values of receptor and ligand density, the rate-limiting density will be identified as the most important parameter. As a result, the importance of integrin density also points to the importance of fibronectin density in modulating the magnitude and kinetics of ERKPP.

3.4.2 One-at-a-time parameter variation: In order to gauge the robustness of the model to changes in parameters, one-at-a-time parameter variations were performed when holding every other parameter fixed at their assumed values. Parameters were varied over three orders of magnitude above and below their assumed values, and the range over which the conclusions drawn in Section 3 held qualitatively was determined (Fig. 6). The model is found to be robust and tolerant of significant changes in many parameters. Parameters $24-64$ and $85-105$ refer to reactions involved in Src activation, She phosphorylation and recruitment of both Grb2 and Sos. Even many of the MAP kinase cascade reaction rates, including some that were identified as the key regulators by Morris screening (ex. 81, 79, 74, 83,82 and so on), do not limit the qualitative conclusions drawn from the model.

\section{Discussion}

This model for the integrin activation of ERK successfully replicated experimentally observed behaviour for FAK and ERK activation, as well as changes in ERK activation in response to fibronectin density. Application of the Morris method to the model allowed the identification of the key parameters contributing to ERKPP dynamics. Thus, the model serves as a tool for understanding and identifying characteristics of the pathway that contribute to different ERKPP behaviour. The insights gained by such models are important steps to ultimately controlling signalling pathways in vivo. 
Table 1: Experimentally controllable parameters identified by Morris screening within the top 85th percentile of parameters important for ERK activation

\begin{tabular}{|c|c|}
\hline ERKPP behaviour & $\begin{array}{l}\text { Experimentally controllable } \\
\text { parameters within 85th } \\
\text { percentile }\end{array}$ \\
\hline overall time course & $\begin{array}{l}\text { PP3 (2), ERK (3), PP2 (7), MEK (8), } \\
\text { PP1 (9), Raf (12), Rt (15), Ras } \\
\text { (16) }\end{array}$ \\
\hline time to $\max$ & $\begin{array}{l}\text { RasGAP (1), Ras (2), MEK (3), Raf } \\
\text { (4), CA (5), PP2 (6), Rt (7), FAK } \\
\text { (11), Grb2 (16) }\end{array}$ \\
\hline$t_{1}=1500 \mathrm{~s}$ & $\begin{array}{l}\text { PP3 (2), ERK (3), PP2 (5), Raf (7), } \\
\text { PP1 (9), Grb2 (11), MEK (14) }\end{array}$ \\
\hline$t_{2}=4000 \mathrm{~s}$ & $\begin{array}{l}\operatorname{PP3}(2), \operatorname{ERK}(3), \operatorname{PP} 2(5), \operatorname{MEK}(8), \\
\text { Ras (10), Rt (11), PP1 (13) }\end{array}$ \\
\hline$t_{3}=15000 \mathrm{~s}$ & $\begin{array}{l}\operatorname{ERK}(2), \operatorname{PP} 3(4), \operatorname{MEK}(6), \operatorname{PP} 2 \text { (7), } \\
\text { PP1 (10) }\end{array}$ \\
\hline maximum value & $\begin{array}{l}\text { PP3 (2), ERK (3), PP2 (6), PP1 (8), } \\
\text { Grb2 (10), MEK (11), Rt (12) }\end{array}$ \\
\hline
\end{tabular}

Each parameter's rank out of the total 106 parameters is included in parentheses. PP1, PP2 and PP3 refer to phosphatases for Raf, MEK and ERK, respectively. CA denotes contact area

The ability of the model to replicate experimentally measured time courses for FAK and ERK in response to adhesion on fibronectin is an important foundation for understanding the adhesion-specific parameters that might contribute to ERK activation. The appearance of an early peak for ERKPP is not surprising as long as there is an excess of FAK, which serves as an early stimulus for ERKPP. Factors affecting FAKP magnitude, such as fibronectin density, are found to be stronger regulators of ERKPP dynamics than those affecting only FAKP kinetics. However, the effects of ligand density are not solely dependent on the initial rate of FAK activation, but are likely transduced downstream by additional pathways. Although additional Western blot time courses have been published, we chose a semi-quantitative time course for the purpose of comparing to simulated results. Deviations between the experimental data used here and other available data may be due to a myriad of differences including cell type and experimental procedure.

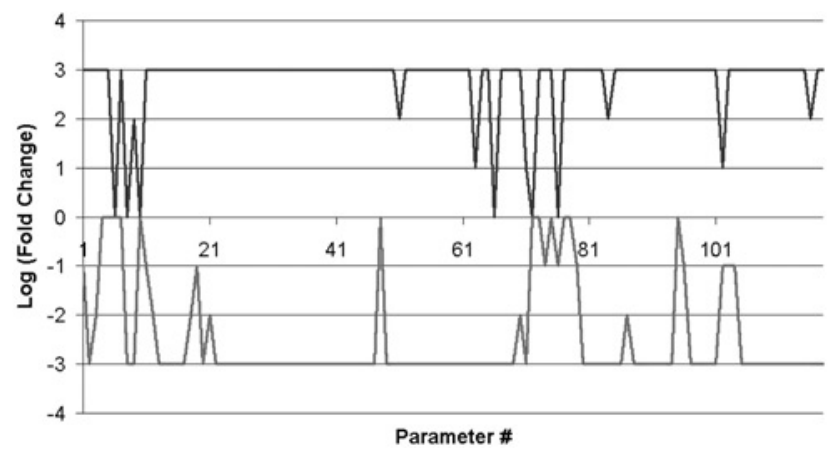

Fig. 6 Parameter ranges over which qualitative results hold

Parameters were varied one at a time over six orders of magnitude when holding all other fixed

Changes in parameter are expressed as $\log _{10}$ of the fold change

Parameters that correspond to each parameter number along the $x$-axis are listed in the Supplementary Materials
The simplified integrin clustering model used is shown to be sufficient for predicting the effects of ligand density on ERK activation. The model based on dimerisation predicts that increases in ligand density will increase the magnitude of FAK activation, which has been shown by Garcia and Boettiger [46]. Changes in the magnitude of FAK activation are then translated into changes in the kinetics and magnitude of ERK activation. Although $k_{\mathrm{p}}$ and $k_{\mathrm{m}}$ may alter the time at which FAK activation reaches its maximum, the ultimate magnitude, as specified by ligand density, will not be affected. Changes in $k_{1}$ may also affect the magnitude of FAK activation, but the relative changes that result from ligand density variations will remain the same. More generally, the magnitude of FAK activation increases with ligand density regardless of the assumed value of $k_{1}$ or $k_{\mathrm{p}}$ and $k_{\mathrm{m}}$. As a result, changes in downstream signalling that originate with ligand density will also remain the same qualitatively. Future work should include a more detailed model of the integrin clustering process so that the roles of additional parameters involved in clustering can be tested.

Raf and Ras were identified as the key regulators of ERK signalling magnitude and duration, in agreement with the control analysis performed by Hornberg et al. [19]. Furthermore, the behaviour of proteins involved in RasGDP/GTP cycling reveals that Raf and Ras may function as a key checkpoint in the MAPK cascade because of the relative kinetics of RasGDP/GTP cycling and RasRaf binding, which allow for the formation of transient and sustained ERKPP signals. However, the main effects of the Raf oncogene may not be mediated by this particular characteristic of the signalling pathway, as was suggested by Hornberg. Increases in [Raf] result in increasing magnitudes of ERK activation, but increasingly transient activation signals. For cell-cycle progression and uncontrolled growth, one would expect that increasing the levels of oncogenic Raf would instead lead to sustained signalling. On the other hand, this model shows that increases in the level of the oncogene Ras result in more sustained ERK signals, although larger changes in the Ras concentration are required. This is consistent with the role of Ras as an oncogene that promotes tumorigenic behaviour. Moreover, the

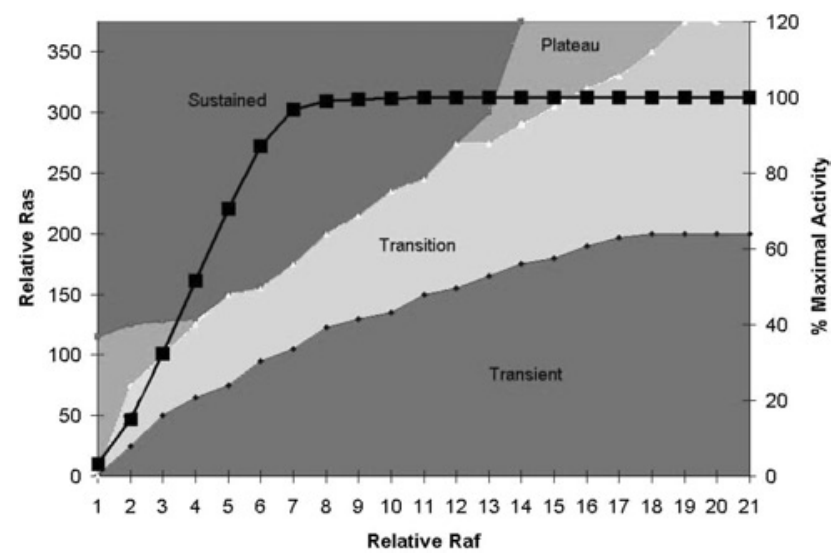

Fig. 7 Effects of Raf and Ras concentrations on ERK activity

Concentrations of Ras and Raf are expressed as multiples of the minimum plotted values $\left(5 \times 10^{-7}\right.$ and $1 \times 10^{-8} \mathrm{M}$, respectively) Magnitude of ERK, expressed as a percentage of the maximal ERK activation, is also plotted for different values of $\operatorname{Raf}(\mathbf{\square})$

Shaded regions represent an initial transient activation followed by sustained mid-level signalling at $33-66 \%$ of maximum (sustained), sustained high-level signaling at $66-100 \%$ of maximum (plateau), a decrease to less than $33 \%$ of maximal signal within $6 \mathrm{~h}$ (transient) or a decrease to less than $33 \%$ of maximum between 6 and $12 \mathrm{~h}$ (transition) 
behaviour of the Raf/Ras checkpoint suggests that mutations in Ras that affect the GDP/GTP cycling, such as its affinity for GAPs or GTPases, may be a mechanism for modulating cell behaviour. Therefore the oncogenic effects of Raf may be mediated mainly by changes in the magnitude of ERK activation, whereas the effects of Ras are more likely to involve changes in signal duration.

This is consistent with Roovers and Assoian's [5] suggestion that cell-cycle progression requires a large transient ERK activation that will induce p21cip expression in the early G1 phase followed by a sustained mid-level activation that promotes cyclin D1 expression and stops the induction of p21cip by late G1 phase. It has also been shown that p21cip is induced by high levels of Raf [47, 48]. Our results would suggest that an ideal combination of Raf and Ras levels could create cell-growth-promoting ERK activities. To determine these levels, Raf and Ras concentrations were varied and their effects on the magnitude and duration of ERK activation were observed (Fig. 7). The concentrations plotted are model-specific, however the trends hold regardless of the parameter values. Increasing Ras concentration promotes transition of ERK activity from transient to sustained behaviour. Here, transient and sustained behaviour refer to initial ERK activation that falls to less than a one-third and between one-third and two-thirds of initial activation, respectively. At low [Raf], a plateau in ERK activation may form in addition to transient and sustained behaviour for certain [Ras]. Also, the magnitude of ERK activity is found to be significantly decreased with [Raf] so that cell-cycle progression might be inhibited regardless of the signal duration. When both Raf and Ras concentrations are high, ERK activation exhibited a large magnitude initial peak that fell off to sustained mid-level activation. This behaviour is consistent with cell-cycle progression. This may correspond to cells in which both Raf and Ras oncogenes are over-expressed or contain activating mutations. Thus, both Raf and Ras may act together to promote cell growth. Raf contributes to the magnitude of the signal, bringing the initial transient activation of ERK to levels that allow for induction of p21cip, whereas Ras primarily extends the duration of ERK, sustaining mid-level activation for long times.

It has been suggested that the peak in ERK activation arises from negative feedback, which turns off the ERK activation signal [49-51]. Feedback, either positive or negative, has not been included in this system. Although negative feedback may occur, we have shown that the $\mathrm{Raf} /$ Ras checkpoint can also play a role in regulating signal duration, particularly in controlling the fall-off of the ERK activation signal. In fact, for certain Raf and Ras concentrations, the transient activity of ERK decreases rapidly, but does not approach zero, instead remaining at some lower level of ERK activation. In such cases, a negative feedback might be an important mechanism for shutting down the remaining ERK activity.

Src and Shc represent parallel pathways by which the MAPK cascade can be activated by FAK. As the model is run now, the Shc pathway dominates signalling to ERK. It is possible for Src to be the main contributor to ERK activation, but that assumption is not explored in detail in this paper. However, the overall effects seen in this paper were confirmed in a pathway in which the levels of Shc are significantly reduced so as to allow the Src pathway to dominate (data not shown).

Although our model is robust and sufficient for predicting ERK activation by adhesion, a complete model of ERK activation should take into account the contributions of both growth factors and adhesion. Cross-talk between growth factor and adhesion signalling is vital for producing highmagnitude, sustained ERK activation. In this paper, a model for adhesion-based activation is presented. Future work will involve the addition of the growth-factor-mediated pathway. Coupled with the detailed model of clustering described above, the model not only complements experimental systems in which signalling in adhesive, growth factor stimulated cells is measured, but also a means for exploring the cross-talk between growth factors and receptors.

\section{Acknowledgments}

This work was supported by a grant from Department of Defense W81XWH-05-1-330 to D.A. Hammer and V.M. Weaver.

\section{References}

1 Hanahan, D., and Weinberg, R.A.: 'The hallmarks of cancer', Cell, 2000, 100, (1), pp. 57-70

2 Aplin, A.E., and Juliano, R.L.: 'Regulation of nucleocytoplasmic trafficking by cell adhesion receptors and the cytoskeleton', J. Cell Biol., 2001, 155, (2), pp. 187-191 [Epub 15 October 2001]

3 Shaulian, E., and Karin, M.: 'AP-1 in cell proliferation and survival', Oncogene, 2001, 20, (19), pp. 2390-2400

4 Marshall, C.: 'How do small GTPase signal transduction pathways regulate cell cycle entry?', Curr. Opin. Cell Biol., 1999, 11, (6), pp. $732-736$

5 Roovers, K., and Assoian, R.K.: 'Integrating the MAP kinase signal into the G1 phase cell cycle machinery', Bioessays, 2000, 22, (9), pp. $818-826$

6 Danen, E.H., and Yamada, K.M.: 'Fibronectin, integrins, and growth control', J. Cell Physiol., 2001, 189, (1), pp. 1-13

7 Renshaw, M.W., Ren, X.D., and Schwartz, M.A. 'Growth factor activation of MAP kinase requires cell adhesion', EMBO J., 1997, 16, (18), pp. 5592-5599

8 Giancotti, F.G., and Ruoslahti, E.: 'Integrin signaling', Science, 1999, 285, (5430), pp. 1028-1032

9 Barberis, L., Wary, K.K., Fiucci, G., Liu, F., Hirsch, E., Brancaccio, M., Altruda, F., Tarone, G., and Giancotti, F.G.: 'Distinct roles of the adaptor protein Shc and focal adhesion kinase in integrin signaling to ERK', J. Biol. Chem., 2000, 275, (47), pp. 36532-36540

10 Schlaepfer, D.D., and Hunter, T.: 'Integrin signalling and tyrosine phosphorylation: just the FAKs?', Trends Cell Biol., 1998, 8, (4), pp. $151-157$

11 Wary, K.K., Mainiero, F., Isakoff, S.J., Marcantonio, E.E., and Giancotti, F.G.: 'The adaptor protein Shc couples a class of integrins to the control of cell cycle progression', Cell, 1996, 87, (4), pp. 733-743

12 Assoian, R.K., and Schwartz, M.A.: 'Coordinate signaling by integrins and receptor tyrosine kinases in the regulation of G1 phase cell-cycle progression', Curr. Opin. Genet. Dev., 2001, 11, (1), pp. 48-53

13 Giancotti, F.G.: 'Integrin signaling: specificity and control of cell survival and cell cycle progression', Curr. Opin. Cell Biol., 1997, 9, (5), pp. 691-700

14 Schwartz, M.A., and Ginsberg, M.H.: 'Networks and crosstalk: integrin signalling spreads', Nat. Cell Biol., 2002, 4, (4), pp. E65-E68

15 Roovers, K., Davey, G., Zhu, X., Bottazzi, M.E., and Assoian, R.K.: 'Alpha5betal integrin controls cyclin D1 expression by sustaining mitogen-activated protein kinase activity in growth factor-treated cells', Mol. Biol. Cell, 1999, 10, (10), pp. 3197-3204

16 Kholodenko, B.N., Demin, O.V., Moehren, G., and Hoek, J.B.: 'Quantification of short term signaling by the epidermal growth factor receptor', J. Biol. Chem., 1999, 274, (42), pp. 30169-30181

17 Schoeberl, B., Eichler-Jonsson, C., Gilles, E.D., and Muller, G.: 'Computational modeling of the dynamics of the MAP kinase cascade activated by surface and internalized EGF receptors', Nat. Biotechnol., 2002, 20, (4), pp. 370-375

18 Chapman, S., and Asthagiri, A.R.: 'Resistance to signal activation governs design features of the MAP kinase signaling module', Biotechnol. Bioeng., 2004, 85, (3), pp. 311-322

19 Hornberg, J.J., Binder, B., Bruggeman, F.J., Schoeberl, B., Heinrich, R., and Westerhoff, H.V.: 'Control of MAPK signalling: from complexity to what really matters', Oncogene, 2005, 24, (36), pp. $5533-5542$ 
20 Asthagiri, A.R., Nelson, C.M., Horwitz, A.F., and Lauffenburger, D.A.: 'Quantitative relationship among integrin-ligand binding, adhesion, and signaling via focal adhesion kinase and extracellular signal-regulated kinase 2', J. Biol. Chem., 1999, 274, (38), pp. 27119-27127

21 Morris, M.D.: 'Factorial sampling plans for preliminary computational experiments', Technometrics, 1991, 33, (2), pp. $161-174$

22 Giannone, G., Dubin-Thaler, B.J., Dobereiner, H.G., Kieffer, N., Bresnick, A.R., and Sheetz, M.P.: 'Periodic lamellipodial contractions correlate with rearward actin waves', Cell, 2004, 116, (3), pp. 431-443

23 Bell, G.I.: 'Models for the specific adhesion of cells to cells', Science, 1978, 200, (4342), pp. 618-627

24 Parsons, J.T.: 'Focal adhesion kinase: the first ten years', J. Cell Sci. 2003, 116, (8), pp. 1409-1416

25 Aplin, A.E., Howe, A., Alahari, S.K., and Juliano, R.L.: 'Signal transduction and signal modulation by cell adhesion receptors: the role of integrins, cadherins, immunoglobulin-cell adhesion molecules, and selectins', Pharmacol. Rev., 1998, 50, (2), pp. $197-263$

26 Kaplan, K.B., Bibbins, K.B., Swedlow, J.R., Arnaud, M., Morgan, D.O., and Varmus, H.E.: 'Association of the amino-terminal half of $\mathrm{C}$-Src with focal adhesions alters their properties and is regulated by phosphorylation of tyrosine-527', EMBO J., 1994, 13, (20), pp. $4745-4756$

27 Bjorge, J.D., Jakymiw, A., and Fujita, D.J.: 'Selected glimpses into the activation and function of Src kinase', Oncogene, 2000, 19, (49), pp. $5620-5635$

28 Boerner, R.J., Kassel, D.B., Barker, S.C., Ellis, B., DeLacy, P., and Knight, W.B.: 'Correlation of the phosphorylation states of pp60c-src with tyrosine kinase activity: the intramolecular pY530-SH2 complex retains significant activity if Y419 is phosphorylated', Biochemistry, 1996, 35, (29), pp. 9519-9525

29 Cole, P., Burn, P., Takacs, B., and Walsh, C.: 'Evaluation of the catalytic mechanism of recombinant human Csk (C-terminal Src kinase) using nucleotide analogs and viscosity effects', J. Biol. Chem., 1994, 269, (49), pp. 30880-30887

30 Schlaepfer, D.D., and Hunter, T.: 'Focal adhesion kinase overexpression enhances ras-dependent integrin signaling to ERK2/ mitogen-activated protein kinase through interactions with and activation of c-Src', J. Biol. Chem., 1997, 272, (20), pp. 13189-13195

31 Schlaepfer, D.D., Jones, K.C., and Hunter, T.: 'Multiple Grb2-mediated integrin-stimulated signaling pathways to ERK2/ mitogen-activated protein kinase: summation of both c-Src- and focal adhesion kinase-initiated tyrosine phosphorylation events', Mol. Cell Biol., 1998, 18, (5), pp. 2571-2585

32 Downward, J.: 'The GRB2/Sem-5 adaptor protein', FEBS Lett., 1994, 338, (2), pp. 113-117

33 Howe, A.K., and Juliano, R.L.: 'Distinct mechanisms mediate the initial and sustained phases of integrin-mediated activation of the Raf/MEK/mitogen-activated protein kinase cascade', J. Biol. Chem., 1998, 273, (42), pp. 27268-27274

34 Wary, K.K., Mariotti, A., Zurzolo, C., and Giancotti, F.G.: 'A requirement for caveolin-1 and associated kinase Fyn in integrin signaling and anchorage-dependent cell growth', Cell, 1998, 94, (5) pp. $625-634$

35 Ladbury, J.E., Lemmon, M.A., Zhou, M., Green, J., Botfield, M.C. and Schlessinger, J.: 'Measurement of the binding of tyrosyl phosphopeptides to SH2 domains: a reappraisal', Proc. Natl Acad. Sci. USA, 1995, 92, (8), pp. 3199-3203

36 Payne, G., Shoelson, S.E., Gish, G.D., Pawson, T., and Walsh, C.T.: 'Kinetics of p56lck and p60src Src homology 2 domain binding to tyrosine-phosphorylated peptides determined by a competition assay or surface plasmon resonance', Proc. Natl Acad. Sci. USA, 1993, 90, (11), pp. 4902-4906

37 Kaneko, T., Kumasaka, T., Ganbe, T., Sato, T., Miyazawa, K., Kitamura, N., and Tanaka, N.: 'Structural insight into modest binding of a non-PXXP ligand to the signal transducing adaptor molecule-2 Src homology 3 domain', J. Biol. Chem., 2003, 278, (48), pp. 48162-48168 [Epub 16 September 2003]

38 Matsuda, M., Ota, S., Tanimura, R., Nakamura, H., Matuoka, K., Takenawa, T., Nagashima, K., and Kurata, T.: 'Interaction between the amino-terminal SH3 domain of $\mathrm{CRK}$ and its natural target proteins', J. Biol. Chem., 1996, 271, (24), pp. 14468-14472

39 Fincham, V.J., Unlu, M., Brunton, V.G., Pitts, J.D., Wyke, J.A., and Frame, M.C.: 'Translocation of Src kinase to the cell periphery is mediated by the actin cytoskeleton under the control of the Rho family of small G proteins', J. Cell Biol., 1996, 135, (6, Pt 1), pp. $1551-1564$

40 Wang, D., Esselman, W.J., and Cole, P.A.: 'Substrate conformational restriction and CD45-catalyzed dephosphorylation of tail tyrosine-phosphorylated Src protein', J. Biol. Chem., 2002, 277, (43), pp. 40428-40433

41 Wang, J., and Walsh, C.T.: 'Mechanistic studies on full length and the catalytic domain of the tandem $\mathrm{SH} 2$ domain-containing protein tyrosine phosphatase: analysis of phosphoenzyme levels and Vmax stimulatory effects of glycerol and of a phosphotyrosyl peptide ligand', Biochemistry, 1997, 36, (10), pp. 2993-2999

42 Zhang, Z.: 'Kinetic and mechanistic characterization of a mammalian protein-tyrosine phosphatase, PTP1', J. Biol. Chem., 1995, 270, (19), pp. 11199-11204

43 Sydor, J.R., Engelhard, M., Wittinghofer, A., Goody, R.S., and Herrmann, C.: 'Transient kinetic studies on the interaction of Ras and the Ras-binding domain of c-Raf-1 reveal rapid equilibration of the complex', Biochemistry, 1998, 37, (40), pp. $14292-14299$

44 Hecker, T.P., Ding, Q., Rege, T.A., Hanks, S.K., and Gladson, C.L.: 'Overexpression of FAK promotes Ras activity through the formation of a $\mathrm{FAK} / \mathrm{p} 120 \mathrm{RasGAP}$ complex in malignant astrocytoma cells', Oncogene, 2004, 23, (22), pp. 3962-3971

45 Sharma, S.V.: 'Rapid recruitment of p120RasGAP and its associated protein, p190RhoGAP, to the cytoskeleton during integrin mediated cell-substrate interaction', Oncogene, 1998, 17, (3), pp. 271-281

46 Garcia, A.J., and Boettiger, D.: 'Integrin-fibronectin interactions at the cell-material interface: initial integrin binding and signaling', Biomaterials, 1999, 20, (23-24), pp. 2427-2433

47 Sewing, A., Wiseman, B., Lloyd, A.C., and Land, H.: 'High-intensity Raf signal causes cell cycle arrest mediated by p21Cip1', Mol. Cell. Biol., 1997, 17, (9), pp. 5588-5597

48 Woods, D., Parry, D., Cherwinski, H., Bosch, E., Lees, E., and McMahon, M.: 'Raf-induced proliferation or cell cycle arrest is determined by the level of Raf activity with arrest mediated by p21Cip1', Mol. Cell. Biol., 1997, 17, (9), pp. 5598-5611

49 Dougherty, M.K., Muller, J., Ritt, D.A., Zhou, M., Zhou, X.Z., Copeland, T.D., Conrads, T.P., Veenstra, T.D., Lu, K.P., and Morrison, D.K.: 'Regulation of raf-1 by direct feedback phosphorylation', Mol. Cell, 2005, 17, (2), pp. 215-224

50 Sundberg-Smith, L.J., Doherty, J.T., Mack, C.P., and Taylor, J.M.: 'Adhesion stimulates direct PAK1/ERK2 association and leads to ERK-dependent PAK1 Thr(212) phosphorylation', J. Biol. Chem., 2005, 280, (3), pp. 2055-2064

51 Brummer, T., Naegele, H., Reth, M., and Misawa, Y.: 'Identification of novel ERK-mediated feedback phosphorylation sites at the C-terminus of B-Raf', Oncogene, 2003, 22, (55), pp. $8823-8834$ 